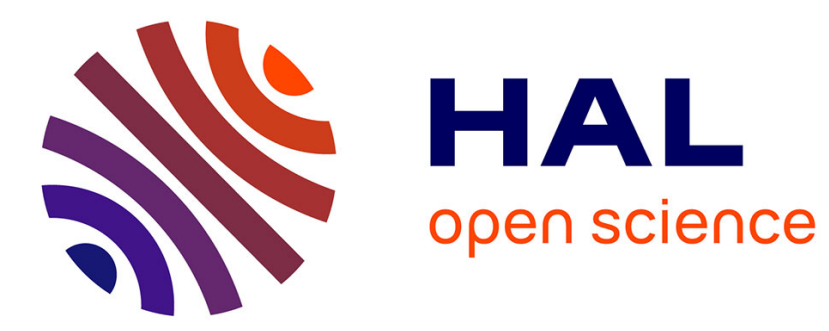

\title{
Étude de niveaux de spin élevé de 13360Nd 73
}

\author{
Y. Choquer, J. Gizon, A. Gizon
}

\section{To cite this version:}

Y. Choquer, J. Gizon, A. Gizon. Étude de niveaux de spin élevé de 13360Nd 73. Journal de Physique Lettres, 1977, 38 (7), pp.157-160. 10.1051/jphyslet:01977003807015700 . jpa-00231349

\section{HAL Id: jpa-00231349 https://hal.science/jpa-00231349}

Submitted on 1 Jan 1977

HAL is a multi-disciplinary open access archive for the deposit and dissemination of scientific research documents, whether they are published or not. The documents may come from teaching and research institutions in France or abroad, or from public or private research centers.
L'archive ouverte pluridisciplinaire HAL, est destinée au dépôt et à la diffusion de documents scientifiques de niveau recherche, publiés ou non, émanant des établissements d'enseignement et de recherche français ou étrangers, des laboratoires publics ou privés. 
Classification

Physics Abstracts

4.118

\title{
ÉTUDE DE NIVEAUX DE SPIN ÉLEVÉ DE ${ }_{60}^{133} \mathrm{Nd}_{73}$
}

\author{
Y. CHOQUER, J. GIZON et A. GIZON \\ Institut des Sciences Nucléaires, B.P. no 257, 38044 Grenoble Cedex, France
}

(Reçu le 4 février 1977, accepté le 28 février 1977)

\begin{abstract}
Résumé. - Les niveaux de spin élevé de ${ }^{133} \mathrm{Nd}$ ont été excités par la réaction ${ }^{116} \mathrm{Sn}\left({ }^{20} \mathrm{Ne}, 3 \mathrm{n}\right){ }^{133} \mathrm{Nd}$ et étudiés par spectroscopie gamma en ligne. Le schéma de niveaux partiel établi contient une structure en bande à parité négative basée sur un niveau $9 / 2^{-}$et associée à un neutron impair de la couche $h_{11 / 2}$.
\end{abstract}

Abstract. - High-spin states in ${ }^{133} \mathrm{Nd}$ have been produced by the reaction ${ }^{116} \mathrm{Sn}\left({ }^{20} \mathrm{Ne}, 3 \mathrm{n}\right){ }^{133} \mathrm{Nd}$ and studied by in-beam $\gamma$-ray spectroscopic techniques. The partial level scheme deduced shows a negative parity band structure based upon a $9 / 2^{-}$state and associated with an odd neutron in the $h_{11 / 2}$ shell.

Les structures des états de spin élevé identifiés à l'aide de réactions (IL, $x \mathrm{n} \gamma$ ) ont fourni récemment de nombreux renseignements sur les déformations des noyaux de masse impaire déficients en neutrons de la région $A \simeq 130$. Ainsi, les bandes découplées observées dans les isotopes de masse impaire de La [1] et les bandes de rotation à parité négative identifiées dans plusieurs isotopes de masse impaire de ${ }_{56} \mathrm{Ba},{ }_{58} \mathrm{Ce}$ et ${ }_{60} \mathrm{Nd}[2,3]$ ont permis d'établir expérimentalement l'existence d'une forme prolate dans plusieurs noyaux de cette zone de transition.

Une interprétation théorique simple des premiers résultats a été donnée par $F$. Stephens [4] avec un couplage rotation-particule aux faibles déformations. Une extension du modèle, avec un cœur triaxial à déformation statique [5], a été employée avec succès par J. Meyer-Ter-Vehn, pour des noyaux de masse impaire $A \simeq 190$ et constitue une approche théorique que l'on peut envisager dans d'autres régions de transition. En effet, les calculs faits dans le cas de ${ }^{129} \mathrm{Ba}[6]$ indiquent un assez bon accord avec l'expérience mais des écarts subsistent dans les valeurs des rapports d'embranchements.

Si l'hypothèse de base d'un cœur triaxial à déformation statique est discutable, les prédictions du modèle constituent un outil très utile pour l'expérimentateur qui recherche une organisation à l'intérieur des structures complexes observées. Seules des études systématiques précises sur plusieurs isotopes d'une même région de transition permettront d'accumuler des connaissances et de développer une interprétation valable.

C'est dans cette perspective que l'étude du noyau inconnu ${ }_{60}^{133} \mathrm{Nd}_{73}$ a été entreprise.
Les expériences ont été réalisées sur le cyclotron à énergie variable de Grenoble, à l'aide de la réaction ${ }_{50}^{116} \mathrm{Sn}\left({ }^{20} \mathrm{Ne}, 3 \mathrm{n}\right){ }_{60}^{133} \mathrm{Nd}$. Nous avons utilisé une cible d'étain de $2 \mathrm{mg} / \mathrm{cm}^{2}$ enrichie à $97,5 \%$ en ${ }^{116} \mathrm{Sn}$ et à l'arrière de laquelle nous avons évaporé $25 \mu \mathrm{m}$ de plomb. Les spectres gamma ont été enregistrés avec divers détecteurs $\mathrm{Ge}(\mathrm{Li})$, soit plan $(R \simeq 0,7 \mathrm{keV}$ à $122 \mathrm{keV})$ soit coaxiaux $(R \simeq 2,5 \mathrm{keV}$ à $1330 \mathrm{keV})$. Les courbes d'efficacités relatives des détecteurs et les étalonnages en énergie ont été obtenus à l'aide de sources étalons $\left({ }^{182} \mathrm{Ta},{ }^{177 \mathrm{~m}} \mathrm{Lu},{ }^{155} \mathrm{Eu},{ }^{152} \mathrm{Eu},{ }^{133 \mathrm{~m}} \mathrm{Ba}\right.$, $110 \mathrm{~m} A g$ ). Les fonctions d'excitations relatives des diverses transitions ont été établies avec des faisceaux de $\mathrm{Ne}^{5+}$ de 75, 80, 85 et $90 \mathrm{MeV}$. L'identification des réactions avec émission de 2,3 et 4 neutrons est assez difficile à cause de l'absence d'informations sur ${ }^{133} \mathrm{Nd}$, sur la désintégration de ${ }^{133} \mathrm{Nd} \rightarrow{ }^{133} \mathrm{Pr}$ et sur ${ }^{132} \mathrm{Nd}$. Cependant, les périodes de ${ }^{133} \mathrm{Nd}$, ${ }^{133} \operatorname{Pr}\left(T_{1 / 2}=6,5 \mathrm{~m}\right),{ }^{132} \mathrm{Nd}$ et ${ }^{132} \operatorname{Pr}\left(T_{1 / 2}=1,6 \mathrm{~m}\right)$ étant courtes, nous avons dû utiliser aussi les résultats connus sur les désintégrations des chaînes $A=133$ $[7,8]$ et $A=132[9,10]$. Les spectres gamma directs prompts et différés, les distributions angulaires et les coïncidences $\gamma-\gamma$ ont été effectuées à $85 \mathrm{MeV}$, énergie favorisant au mieux l'excitation de ${ }^{133} \mathrm{Nd}$.

L'examen d'une portion de spectre direct (Fig. 1) montre que l'étude de ${ }^{133} \mathrm{Nd}$ est très complexe à cause de nombreuses transitions associées à la radioactivité courte. La comparaison des spectres gamma prompts, différés et de radioactivité pris à des temps variés après coupure du faisceau, a permis d'identifier une trentaine de transitions, d'énergie inférieure à $1,3 \mathrm{MeV}$, qui appartiennent à ${ }^{133} \mathrm{Nd}$. Les distributions angulaires des rayonnements gamma ont été mesurées 


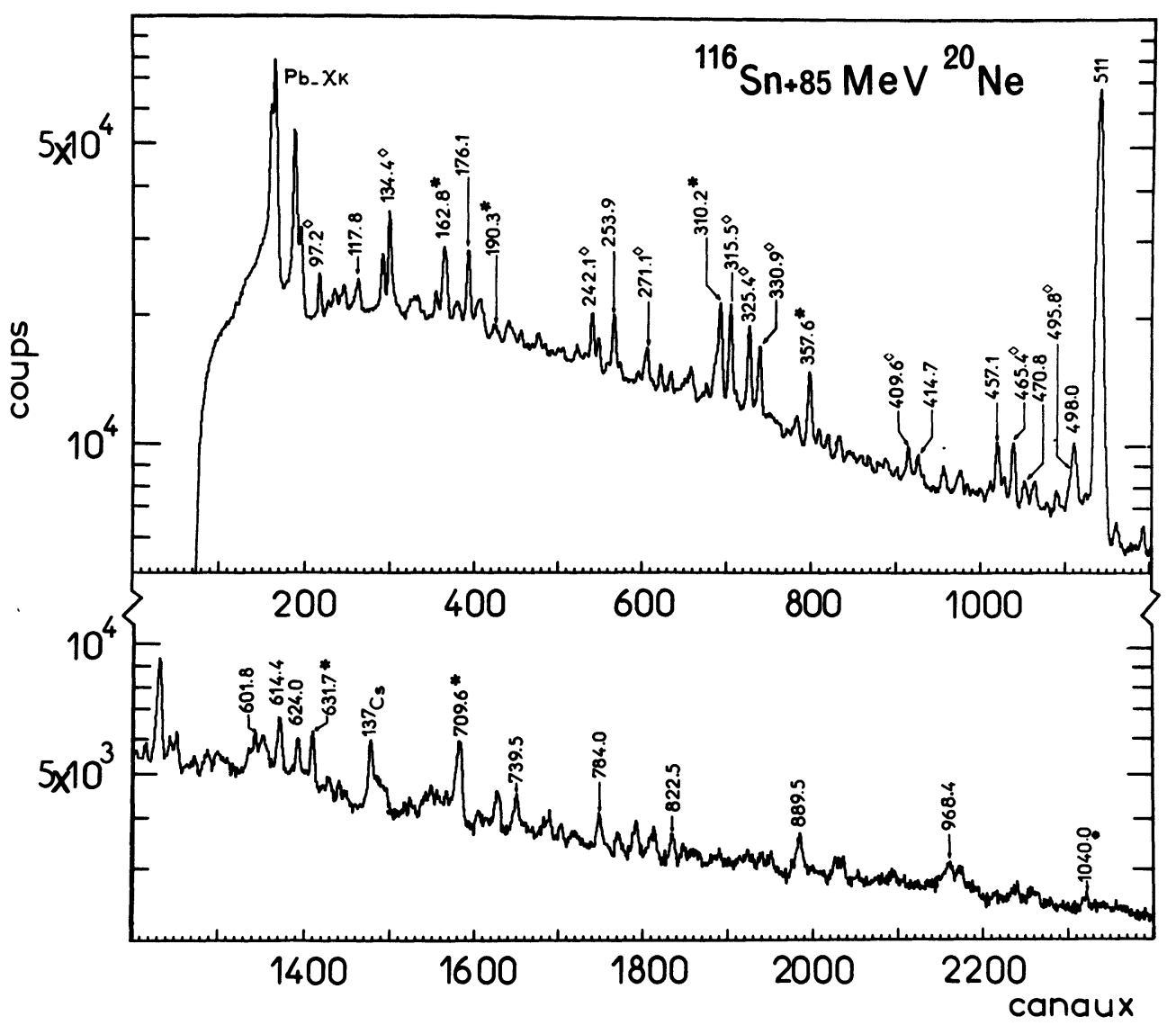

FIG. 1. - Spectre gamma direct produit dans la réaction ${ }^{116} \mathrm{Sn}\left({ }^{20} \mathrm{Ne}, 3 \mathrm{n}\right)$ à $85 \mathrm{MeV}$. Les raies associées à la radioactivité sont indiquées par des losanges. Les astérisques représentent des pics complexes.

[Single $\gamma$-ray spectrum produced in the reaction ${ }^{116} \mathrm{Sn}\left({ }^{20} \mathrm{Ne}, 3 \mathrm{n}\right){ }^{133} \mathrm{Nd}$ at $85 \mathrm{MeV}$. Lines associated with decay are indicated by diamonds. Asterisks show composite peaks.]

TABLEAU I

Transitions gamma de ${ }^{133} \mathrm{Nd}$ produites dans la réaction ${ }^{116} \mathrm{Sn}\left({ }^{20} \mathrm{Ne}, 3 \mathrm{n}\right){ }^{133} \mathrm{Nd}$ et placées dans la bande à parité négative

[Gamma transitions of ${ }^{133} \mathrm{Nd}$ produced in the reaction ${ }^{116} \mathrm{Sn}\left({ }^{20} \mathrm{Ne}, 3 \mathrm{n}\right){ }^{133} \mathrm{Nd}$ and placed in the odd-parity band.]

\begin{tabular}{|c|c|c|c|c|}
\hline$E_{\gamma}$ & $\underline{I_{\gamma}}$ & $A_{2}^{\exp }$ & Multipolarité & Position \\
\hline 162,8 & $81 \pm 10$ & $-0,67 \pm 0,05$ & $\mathbf{M} 1+\mathrm{E} 2$ & $11 / 2^{-} \rightarrow 9 / 2^{-}$ \\
\hline $190,3\left(^{a}\right)$ & $19 \pm 4$ & $-0,34 \pm 0,09$ & $\mathrm{M} 1+\mathrm{E} 2$ & $15 / 2^{-} \rightarrow 13 / 2^{-}$ \\
\hline 307,7 & $41 \pm 8$ & $-0,36 \pm 0,06$ & $\mathrm{M} 1+\mathrm{E} 2$ & $13 / 2^{-} \rightarrow 11 / 2^{-}$ \\
\hline 428,3 & $33 \pm 5$ & $<0$ & $(\mathrm{M} 1+\mathrm{E} 2)$ & $17 / 2^{-} \rightarrow 15 / 2^{-}$ \\
\hline 470,8 & $45 \pm 5$ & $0,06 \pm 0,15$ & (E2) & $13 / 2^{-} \rightarrow 9 / 2^{-}$ \\
\hline 498,0 & 100 & $0,10 \pm 0,07$ & E2 & $15 / 2^{-} \rightarrow 11 / 2^{-}$ \\
\hline 503,6 & $\sim 5$ & & & $(15 / 2)^{-} \rightarrow 13 / 2^{-}$ \\
\hline 618 & $(b)$ & & & $17 / 2^{-} \rightarrow 13 / 2^{-}$ \\
\hline 624,0 & $43 \pm 5$ & $>0$ & (E2) & $19 / 2^{-} \rightarrow 15 / 2^{-}$ \\
\hline $709,6\left(^{a}\right)$ & $<40$ & $>0$ & (E2) & $23 / 2^{-} \rightarrow 19 / 2^{-}$ \\
\hline $770,5\left(^{a}\right)$ & $11 \pm 2$ & $>0$ & (E2) & $(27 / 2)^{-} \rightarrow 23 / 2^{-}$ \\
\hline
\end{tabular}

( ${ }^{a}$ Transition appartenant à un groupe complexe.

(b) Très faible et seulement observée dans les spectres de coïncidences $\gamma-\gamma$. 
à des angles de $0^{\circ}, 55^{\circ}$ et $90^{\circ}$ par rapport à la direction du faisceau incident et fournissent une estimation du coefficient de distribution angulaire $A_{2}^{\exp }$ pour les transitions les plus intenses (Tableau I). Les coïncidences $\gamma-\gamma$ ont été faites avec deux détecteurs coaxiaux de 30 et $77 \mathrm{~cm}^{3}$. Leur analyse a été rendue difficile par la présence de raies intenses associées à la radioactivité courte de la chaîne $A=133$ et de transitions de ${ }^{129} \mathrm{Ce}$ et ${ }^{125} \mathrm{Ba}$ produits par les ions ${ }^{16} \mathrm{O}^{+4}$ et ${ }^{12} \mathrm{C}^{+3}$ contaminant le faisceau de ${ }^{20} \mathrm{Ne}^{+5}$.

Le niveau fondamental et les premiers états excités de ${ }^{133} \mathrm{Nd}$ étant inconnus, l'établissement d'un schéma de niveaux est délicat. Nous avons dû travailler par approches successives en tenant compte des niveaux atteints par réactions (ions lourds, $x \mathrm{n}$ ) dans les isotopes et les isotones de masse impaire voisins. Dans les isotones $N=77$ et $N=75$ de $\mathrm{Nd}, \mathrm{Ba}$ et Ce et dans ceux $N=73$ de Ba et Ce, les réactions du type $\left({ }^{12} \mathrm{C}, x \mathrm{n}\right)$ et $\left({ }^{16} \mathrm{O}, x \mathrm{n}\right)$ sur des cibles appropriées permettent d'exciter fortement des niveaux de haut spin d'une structure en bande, de parité négative, issue d'un trou de neutron $h_{11 / 2}$. En étudiant ${ }^{133} \mathrm{Nd}$ par la réaction ${ }^{116} \mathrm{Sn}\left({ }^{20} \mathrm{Ne}, 3 \mathrm{n}\right){ }^{133} \mathrm{Nd}$ on peut donc s'attendre à trouver une structure analogue.

L'analyse de l'ensemble des données expérimentales permet d'établir dans ${ }^{133} \mathrm{Nd}$ une bande analogue à celle trouvée dans ${ }^{131} \mathrm{Ce}$ et ${ }^{129} \mathrm{Ba}$. D'après les analogies entre énergie et nature des transitions, alimentation et désexcitation des niveaux, cette bande fortement peuplée est assimilée à la structure $h_{11 / 2}$ basée sur l'état $9 / 2^{-}$(Fig. 2).

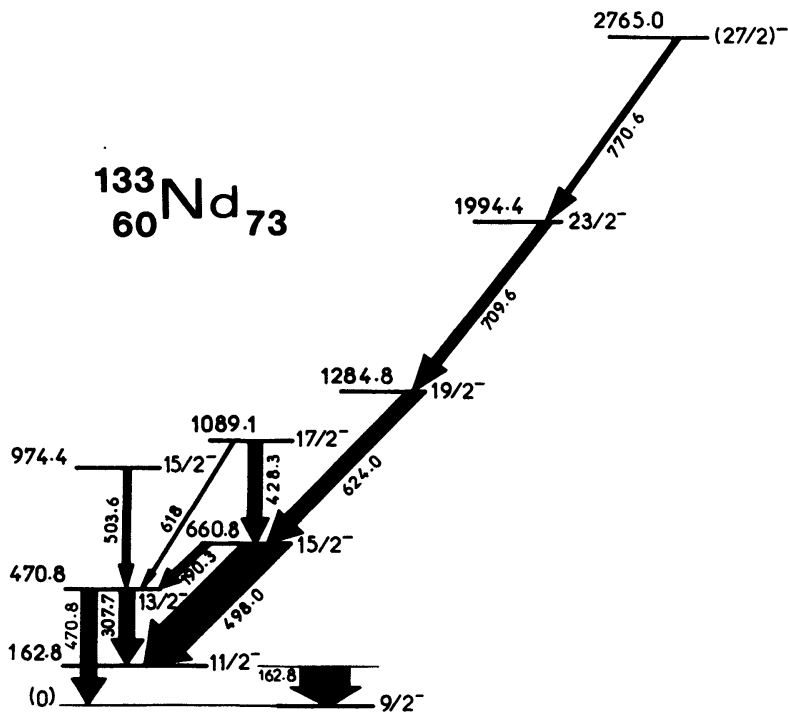

FIG. 2. - Schéma de niveaux partiel de ${ }^{133} \mathrm{Nd}$. La largeur des flèches est proportionnelle à l'intensité totale des transitions.

[Partial level scheme of ${ }^{133} \mathrm{Nd}$. The widths of the arrows are proportional to the total transition intensity.]

Seules les transitions appartenant à la structure $h_{11 / 2}$ sont portées dans le tableau I. Parmi les autres non placées, il n'a pas été possible de mettre en évidence la bande $g_{7 / 2}$ plus faiblement alimentée qui avait déjà été identifiée dans ${ }^{131} \mathrm{Ce}$ et ${ }^{129} \mathrm{Ba}$.
Les données expérimentales actuelles ne permettent pas de dire si l'état de base $9 / 2^{-}$du système $h_{11 / 2}$ est un isomère ou l'état fondamental de ${ }^{133} \mathrm{Nd}$. D'après nos mesures, sa durée de vie est inférieure à 2 minutes. Une identification très récente de ${ }^{133} \mathrm{Nd}$ produit par les réactions ${ }^{102} \mathrm{Nd}+{ }^{32} \mathrm{~S}$ et ${ }^{106} \mathrm{Cd}+{ }^{32} \mathrm{~S}$ et séparé isotopiquement en ligne a permis de déterminer pour cet isotope une période de $(70 \pm 10)$ secondes [11].

Les structures à parité négative observées dans les isotopes de néodyme de masse impaire déficients en neutrons ont été reportées sur la figure 3, en normalisant les énergies sur le niveau $11 / 2^{-}$afin de mettre en évidence l'évolution de la déformation $\beta$ avec la masse des noyaux. Une diminution progressive des écarts entre niveaux apparaît aussi sur la figure 4 où sont regroupés les résultats obtenus pour les isotones $N=73$ de la région. Là encore, ${ }^{133} \mathrm{Nd}$ se présente comme le noyau le plus déformé $\beta$. Ceci peut se justifier par le fait qu'il est le plus éloigné des couches pleines $Z=50$ et $N=82$.

Bien que les informations obtenues sur ${ }^{133} \mathrm{Nd}$ soient incomplètes notamment en ce qui concerne les probabilités de transition, il apparaît que cet isotope s'insère parfaitement parmi les noyaux de masse impaire voisins pour lesquels le modèle à cœur triaxial de J. Meyer-Ter-Vehn [5] constitue une bonne approche théorique. Puisque le paramètre de déformation $\beta$ est déduit des premiers niveaux excités des noyaux pair-pair voisins, nous avons dû déterminer ceux de ${ }^{132} \mathrm{Nd}$ jusqu'alors inconnus. Les expériences
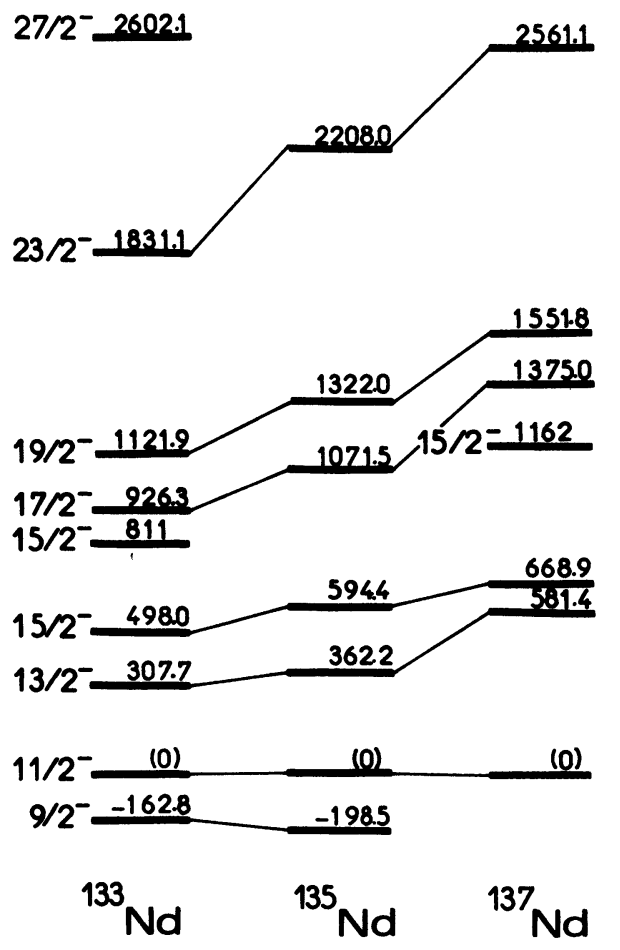

Fig. 3. - Comparaison des structures à parité négative observées dans les isotopes de néodyme.

[Comparison of odd-parity level structures observed in $\mathrm{Nd}$ isotopes.] 

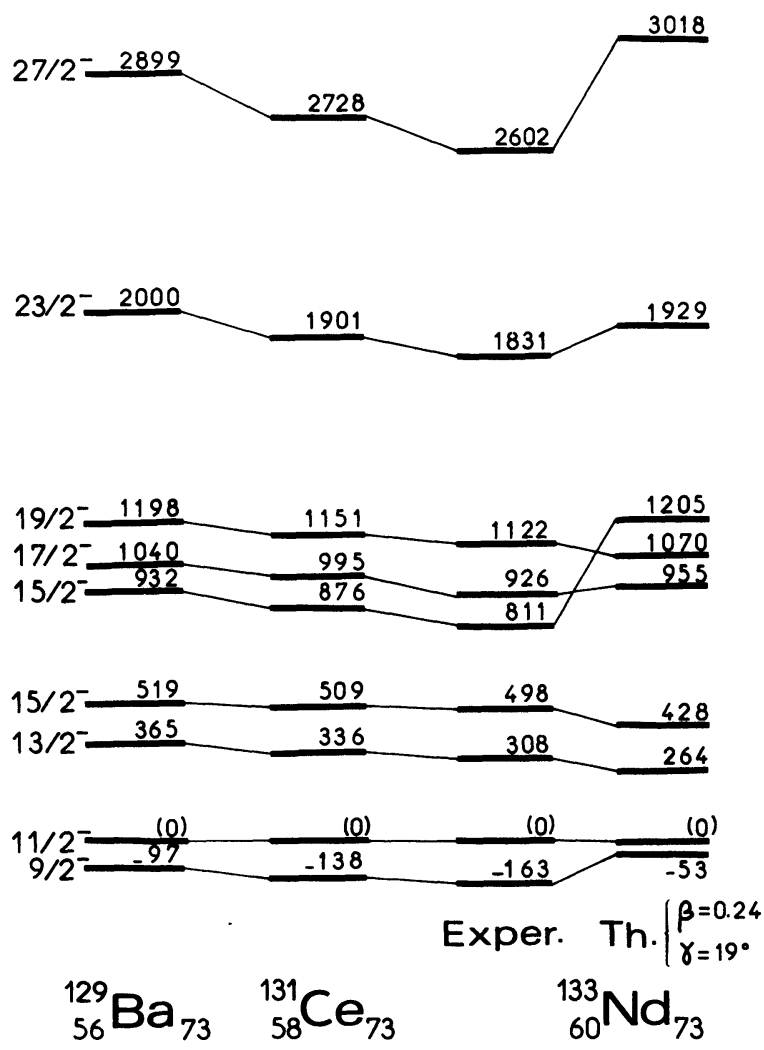

Frg. 4. - Comparaison des structures à parité négative observées dans les isotones $N=73$ voisins de ${ }^{133} \mathrm{Nd}$.

[Comparison of odd-parity level structures observed in $N=73$ isotones near ${ }^{133} \mathrm{Nd}$.] montrent que les niveaux $2^{+}$et $4^{+}$de ${ }^{132} \mathrm{Nd}$ sont respectivement à 283,6 et $741 \mathrm{keV}$. Le couplage d'un trou de neutron $\mathrm{h}_{11 / 2}$ avec un cour triaxial de type allongé ayant les déformations $\beta=+0,24$ et $\gamma=19^{\circ}$ donne le spectre de niveaux calculé représenté sur la figure 4. La surface de Fermi est située entre les orbitales $9 / 2^{-}$et $7 / 2^{-}$de la couche $h_{11 / 2}$.

\section{Bibliographie}

[1] Leigh, J. R., Nakai, K., Maier, K. H., Puhlhoffer, F., Diamond, R. M. and Stephens, F. S., Nucl. Phys. A 213 (1973) 1.

[2] Gizon, J., Gizon, A., Maier, M. R., Diamond, R. M. and Stephens, F. S., Nucl. Phys. A 222 (1974) 557.

[3] Gizon, J., Gizon, A. and Horen, D. J., Nucl. Phys. A 252 (1975) 509.

[4] Stephens, F. S., Rev. Mod. Phys. 47 (1975) 43.

[5] Meyer-Ter-Vehn, J., Nucl. Phys. A 249 (1975) 111 et 141.

[6] Gizon, J., Gizon, A., Meyer-Ter-Vehn, J., Nucl. Phys. in press.

[7] Arlt, R., Beyer, G., Gromov, K. Y., Musiol, G., OrtLEPP, H. G., TYrRofF, H., USMANOVA, Z. A., STRUSNY, H. and HerrmanN, E., J.I.N.R. P6-6285, Dubna (1972).

[8] Henry, E. A., Nucl. Data Sheets 11 (1974) 495.

[9] Latuszynski, A., Ortlepp, H. G. and Yasinski, A., Proc. of the Conf. of Nuclear Physics Dubna (1975) p. 102.

[10] Hiddleston, H. R. and Browne, C. P., Nucl. Data Sheets 17 (1976) 225.

[11] Bogdanov, D. D., Demyanov, A. V., Karnaukhov, V. A., Petrov, L. A., Plohocki, A., Subbotin, V. G. and VoBORIL, J., Nucl. Phys. A 275 (1977) 229. 